\title{
NONLINEARLY PERTURBED RENEWAL EQUATIONS: THE NONPOLYNOMIAL CASE
}

UDC 519.21

\author{
YING NI
}

\begin{abstract}
Models of nonlinearly perturbed renewal equations with nonpolynomial perturbations are studied. Exponential asymptotic expansions are given for the solutions to the perturbed renewal equations under consideration. An application to perturbed $\mathrm{M} / \mathrm{G} / 1 /$ queues is presented.
\end{abstract}

\section{INTRODUCTION}

The object of study is the following model of a perturbed renewal equation which holds for every $\varepsilon \geq 0$ :

$$
x_{\varepsilon}(t)=q_{\varepsilon}(t)+\int_{0}^{t} x_{\varepsilon}(t-s) F_{\varepsilon}(d s), \quad t \geq 0 .
$$

In this model, the forcing function $q_{\varepsilon}(\cdot)$ and the distribution function generating the renewal equation $F_{\varepsilon}(\cdot)$ are assumed to depend on some small perturbation parameter $\varepsilon \geq 0$. In addition, we assume that $q_{\varepsilon}$ and $F_{\varepsilon}$ converge in some natural sense to the limiting functions $q_{0}$ and $F_{0}$ respectively (conditions $\mathbf{A}, \mathbf{C}$ in Section 2), which allows us to consider (11) with $\varepsilon>0$ as a perturbed version of (1) with $\varepsilon=0$. When $\varepsilon=0$, equation (11) is simply reduced to the classical renewal equation. We investigate the case where $F_{\varepsilon}$ can be improper, i.e. $F_{\varepsilon}(\infty) \leq 1$, or in other words the defect $f_{\varepsilon}=1-F_{\varepsilon}(\infty) \geq$ 0 for $\varepsilon \geq 0$. However we restrict the limiting distribution function to be proper; thus $f_{\varepsilon} \rightarrow 0$ as $\varepsilon \rightarrow 0$. The aim is to obtain the asymptotical behavior of the solution $x_{\varepsilon}(t)$ as $t \rightarrow \infty$ and $\varepsilon \rightarrow 0$ simultaneously. Without loss of generality, we can consider $t$ as a function of $\varepsilon$ and write it as $t_{\varepsilon}$.

Let us also impose some Cramér type condition (condition $\mathbf{B}$ in Section 2) on $F_{\varepsilon}$. It is known that in this case we have the following asymptotic relation for the solution $x_{\varepsilon}(t)$ (Silvestrov, 1976, 1978, 1979, [7- 9 ):

$$
e^{\rho_{\varepsilon} t_{\varepsilon}} x_{\varepsilon}\left(t_{\varepsilon}\right) \rightarrow x_{0}(\infty) \text { as } \varepsilon \rightarrow 0, t_{\varepsilon} \rightarrow \infty,
$$

where the normalizing coefficient $\rho_{\varepsilon}$ is the root of some nonlinear characteristic equation for $F_{\varepsilon}$ (equation (4) in Section 2). This asymptotic relation can be viewed as the generalized version of the classical renewal theorem (see Feller 1971, 2]). Relation (2) can be improved if the normalizing coefficient $\rho_{\varepsilon}$ can be expressed in a more explicit form instead of being given implicitly as a root of a nonlinear equation. In a study by Silvestrov $(1995,[10])$, the defect $f_{\varepsilon}$ and moments $m_{\varepsilon r}=\int_{0}^{\infty} s^{r} F_{\varepsilon}(d s), r=1, \ldots, k$, of the distribution function $F_{\varepsilon}$ are assumed to have asymptotical expansions in $\varepsilon$, i.e. (a) $f_{\varepsilon}=b_{1,0} \varepsilon+\cdots+b_{k, 0} \varepsilon^{k}+o\left(\varepsilon^{k}\right)$, (b) $m_{\varepsilon r}=b_{1, r} \varepsilon+\cdots+b_{k-r, r} \varepsilon^{k}+o\left(\varepsilon^{k-r}\right)$ as $\varepsilon \rightarrow 0$.

2010 Mathematics Subject Classification. Primary 60K05, 34E10; Secondary 60K25.

Key words and phrases. Perturbed renewal equation, nonpolynomial perturbation. 
By imposing some balancing condition on how $t \rightarrow \infty$ and $\varepsilon \rightarrow 0$ simultaneously, the corresponding asymptotical expansion of $\rho_{\varepsilon}$ can be obtained with the coefficients determined by the given coefficients in (a), (b). Using this expansion of $\rho_{\varepsilon}$, the asymptotical relation (2) can be improved to an explicit form, namely the exponential asymptotic expansion of $x_{\varepsilon}(t)$.

In the study mentioned above, the characteristics of $F_{\varepsilon}$, namely the defect and moments, are smooth nonlinear functions of infinitesimal $\varepsilon$ so that their expansions consist of integer powers of infinitesimal $\varepsilon$. The present paper deals with a natural generalization of this setting; here the defect and moments are nonlinear functions of multiple infinitesimals $\tilde{\varepsilon}^{\theta_{i}}, i=1, \ldots, m$, where the $\theta_{i}$ are arbitrary real positive numbers. Moreover, these two characteristics can be expanded into asymptotic expansions containing products of integer powers of the infinitesimals, i.e. $\phi_{\vec{n}}(\tilde{\varepsilon})=\prod_{i=1}^{m} \tilde{\varepsilon}^{n_{i} \theta_{i}}, \vec{n}=\left(n_{1}, \ldots, n_{m}\right)$, $n_{1}, \ldots, n_{m} \in \mathbf{N}_{0}$, where $\mathbf{N}_{0}$ is the set of nonnegative numbers. By a suitable transformation of the infinitesimal $\tilde{\varepsilon}$ into another one $\varepsilon=\varepsilon(\tilde{\varepsilon})$, expansions of this type can be reduced into a more convenient formulation as given below.

In this formulation, we consider the case where the defect and moments can be expanded in $\varepsilon$ up to some order $\alpha$ with respect to the following nonpolynomial asymptotic scale:

$$
\left\{\varphi_{\vec{n}}(\varepsilon)=\varepsilon^{\vec{n} \cdot \vec{\omega}}, \vec{n} \in \mathbf{N}_{0}^{k}\right\} \quad \text { as } \varepsilon \rightarrow 0,
$$

where $\mathbf{N}_{0}^{k} \equiv \mathbf{N}_{0} \times \cdots \times \mathbf{N}_{0}, 1 \leq k<\infty$, with the product being taken $k$ times, and $\vec{\omega}$ is a parameter vector of dimension $k$. In (3), $\vec{n} \cdot \vec{\omega}$ denotes the dot product of the vectors $\vec{n}$ and $\vec{\omega}$. By the definition of an asymptotic scale, the gauge functions $\varphi_{\vec{n}}(\varepsilon), \vec{n} \in \mathbf{N}_{0}^{k}$ are ordered by $\vec{n}$ such that $\varphi_{\vec{n}^{\prime \prime}}(\varepsilon)=o\left(\varphi_{\vec{n}^{\prime}}(\varepsilon)\right)$ if $\vec{n}^{\prime} \cdot \vec{\omega}<\vec{n}^{\prime \prime} \cdot \vec{\omega}$ as $\varepsilon \rightarrow 0$. We shall assume throughout the paper that (3) is ordered in this way. Further, we impose the following assumption for the parameter vector $\vec{\omega}=\left(\omega_{1}, \omega_{2}, \ldots, \omega_{k}\right)$ : (i) $1=\omega_{1}<\omega_{2}<\ldots<\omega_{k}$; (ii) the components are linearly independent over the field $\mathbb{Q}$ of rational numbers, i.e., $\omega_{i} / \omega_{j}$ is an irrational number for any $i \neq j, i, j=1, \ldots, k$. It follows obviously from (i) and (ii) that $\omega_{2}, \ldots, \omega_{k}$ are irrational numbers. Throughout the paper, the symbol $\vec{\omega}$ is reserved for some parameter vector satisfying these two properties. Note that $\vec{\omega}$ can be determined from $\theta_{i}, i=1, \ldots, m$, after the transformation mentioned above.

The main result of the present work is an exponential asymptotic expansion of the solution to the model of the perturbed renewal equation (11) where the defect and moments of $F_{\varepsilon}$ have asymptotic expansions in $\varepsilon$ with respect to (3).

For a general theory of nonlinearly perturbed renewal equations with various applications to nonlinearly perturbed stochastic systems, the book by Gyllenberg and Silvestrov (2008, [3]) is the main reference. We refer also to Ni (2010b, 6]) for a survey on the theory and applications of nonlinearly perturbed renewal equations.

\section{Problem Setup}

We consider the perturbed renewal equation (1) which holds for every $\varepsilon \geq 0$. The forcing function $q_{\varepsilon}(t)$ is a measurable, real-valued and locally bounded function on $[0, \infty)$, and the distribution function $F_{\varepsilon}(\cdot)$ on $[0, \infty)$ is not concentrated on 0 and can be improper. It is known that there exists a unique, measurable and locally bounded solution, $x_{\varepsilon}(t)$, to this renewal equation. The defect and moments of $F_{\varepsilon}$ are defined respectively as $f_{\varepsilon}=1-F_{\varepsilon}(\infty)$ and $m_{\varepsilon r}=\int_{0}^{\infty} s^{r} F_{\varepsilon}(d s), r \geq 1$. We use the notation $\Rightarrow$ for the weak convergence of distribution functions and the notation $\varlimsup$ lim and $\underline{\lim }$ for lim sup and lim inf respectively. The following perturbation conditions are imposed on $F_{\varepsilon}(\cdot)$ and $q_{\varepsilon}(\cdot)$.

A: $F_{\varepsilon}(\cdot) \Rightarrow F_{0}(\cdot)$ as $\varepsilon \rightarrow 0$, where $F_{0}(\cdot)$ is a proper nonarithmetic distribution function. 
B: There exists $\delta>0$ such that $\varlimsup_{0 \leq \varepsilon \rightarrow 0} \int_{0}^{\infty} e^{\delta s} F_{\varepsilon}(d s)<\infty$.

C: (a) $\lim _{u \rightarrow 0} \varlimsup_{0 \leq \varepsilon \rightarrow 0} \sup _{|v| \leq u}\left|q_{\varepsilon}(t+v)-q_{0}(t)\right|=0$ almost everywhere with respect to Lebesgue measure on $[0, \infty)$;

(b) $\varlimsup_{0 \leq \varepsilon \rightarrow 0} \sup _{0 \leq t \leq T}\left|q_{\varepsilon}(t)\right|<\infty$ for every $T \geq 0$;

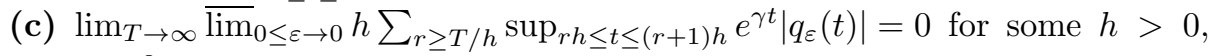
$\gamma>0$.

Under the Cramér type condition, i.e. condition $\mathbf{B}$, the exponential moment of $F_{\varepsilon}$, $\phi_{\varepsilon}(\rho)=\int_{0}^{\infty} e^{\rho s} F_{\varepsilon}(d s), \rho \geq 0$, is finite for $\rho<\delta$ and for $\varepsilon$ small enough. Under conditions $\mathbf{A}$ and $\mathbf{B}$, it is known that there is a unique nonnegative root, $\rho_{\varepsilon}$, of the characteristic equation:

$$
\phi_{\varepsilon}(\rho)=\int_{0}^{\infty} e^{\rho s} F_{\varepsilon}(d s)=1
$$

for $\varepsilon$ small enough and in addition $\rho_{\varepsilon} \rightarrow 0$ as $\varepsilon \rightarrow 0$.

The starting point is the asymptotic relation (2) stated formally as follows.

Theorem 1. Let conditions $\mathbf{A}, \mathbf{B}$ and $\mathbf{C}$ be satisfied. Then for any $0 \leq t_{\varepsilon} \rightarrow \infty$ as $\varepsilon \rightarrow 0$, the following asymptotic relation holds:

$$
\frac{x_{\varepsilon}\left(t_{\varepsilon}\right)}{\exp \left\{-\rho_{\varepsilon} t_{\varepsilon}\right\}} \rightarrow x_{0}(\infty)=\frac{\int_{0}^{\infty} q_{0}(s) d s}{m_{01}} \quad \text { as } \varepsilon \rightarrow 0 .
$$

Note that under condition $\mathbf{A}$ we have $f_{\varepsilon} \rightarrow f_{0}=0$ as $\varepsilon \rightarrow 0$ and under Condition $\mathbf{B}$ all moments of $F_{\varepsilon}$ are finite, i.e. $m_{\varepsilon r}<\infty$ for $r \geq 1$. Conditions $\mathbf{A}$ and $\mathbf{B}$ together imply that for $\varepsilon$ small enough, $m_{\varepsilon r} \rightarrow m_{0 r} \in(0, \infty)$ as $\varepsilon \rightarrow 0$ for $r \geq 1$. We impose also additional perturbation conditions on $f_{\varepsilon}$ and $m_{\varepsilon r}$ in the form of asymptotic expansions with respect to the asymptotic scale (3). To do this we need some additional notation.

For some real number $\alpha \geq 1$ and a given parameter vector $\vec{\omega}$, the notation $[\alpha]_{\vec{\omega}}$ is defined as: $[\alpha]_{\vec{\omega}}=\max (\vec{n} \cdot \vec{\omega}: \vec{n} \cdot \vec{\omega} \leq \alpha)$, where $\vec{n} \in \mathbf{N}_{0}^{k}$. This implies that the last gauge function in (3) that has order less than or equal to $\alpha$ is $\varepsilon^{[\alpha]_{\vec{\omega}}}$. Given $\alpha$ and a specific parameter vector $\vec{\omega}$, by property (ii) of $\vec{\omega}$ we know that there exists a unique vector $\vec{n}$ such that $[\alpha]_{\vec{\omega}}=\vec{n} \cdot \vec{\omega}$; we denote this $\vec{n}$ by $\vec{f}(\alpha, \vec{\omega})$. In particular, this notation is used in Theorem 2 in the next section.

The integer part of a number $\alpha$ is denoted by $[\alpha]$. Let us also define the following two sets:

$$
\mathbf{R}_{i}(\vec{n})=\left\{\vec{p}: p_{1} \leq n_{1}, \ldots, p_{k} \leq n_{k}, \sum_{j=1}^{k} p_{j} \geq i\right\}, \quad \mathbf{R}_{i}^{\prime}(\vec{n})=\mathbf{R}_{i}(\vec{n}) \backslash\{\vec{n}\},
$$

where $\vec{n}, \vec{p} \in \mathbf{N}_{0}^{k}$. For example, when $\vec{n}=(2,1)$ and $i=2, \mathbf{R}_{i}(\vec{n})$ is the set

$$
\{(1,1),(2,0),(2,1)\}
$$

and $\mathbf{R}_{i}^{\prime}(\vec{n})$ the set $\{(1,1),(2,0)\}$.

All vectors are $k$-dimensional (as for $\vec{\omega}$ ) row vectors unless stated otherwise, and they are represented with lowercase Roman/Greek letters with right-pointing arrows above. Symbol $\overrightarrow{0}$ is a vector with all components equal to zeros, and $\vec{e}_{i}$ refers to the $i$ th unit vector, i.e. all components are zero except that the $i$ th component is equal to one.

We are now in a position to impose the following additional perturbation conditions which hold for a given real number $\alpha \geq 1$ and for some given parameter vector $\vec{\omega}$ :

$$
\begin{aligned}
\mathbf{P}_{\vec{\omega}}^{(\alpha)}: & \text { (a) } 1-f_{\varepsilon}=1+\sum_{1 \leq \vec{n} \cdot \vec{\omega} \leq \alpha} b_{\vec{n}, 0} \varepsilon^{\vec{n} \cdot \vec{\omega}}+o\left(\varepsilon^{[\alpha]_{\vec{\omega}}}\right), \\
& \text { (b) } m_{\varepsilon r}=m_{0 r}+\sum_{1 \leq \vec{n} \cdot \vec{\omega} \leq \alpha-r} b_{\vec{n}, r} \varepsilon^{\vec{n} \cdot \vec{\omega}}+o\left(\varepsilon^{[\alpha]_{\vec{\omega}}-r}\right) \text {, for } r=1, \ldots,[\alpha],
\end{aligned}
$$


where all coefficients are finite. In condition $\mathbf{P}_{\vec{\omega}}^{(\alpha)}$, the defect and moments are expanded, up to order $\alpha$, with respect to the asymptotic scale (3). Note that in the trivial case $\vec{\omega}=1$, condition $\mathbf{P}_{\vec{\omega}}^{(\alpha)}$ coincides with the polynomial case, i.e. expansions (a), (b) in Section 1. For convenience, the notation $b_{\overrightarrow{0}, 0}=1, b_{\overrightarrow{0}, r}=m_{0 r}$ is also used.

\section{MAin Results}

The main result of the present paper is given in the following theorem.

Theorem 2. Let conditions $\mathbf{A}, \mathbf{B}$ and $\mathbf{P}_{\vec{\omega}}^{(\alpha)}$ be satisfied. Then:

(i) There exists a unique nonnegative solution to the characteristic equation (4) for all $\varepsilon$ that are small enough. Further, the following expansion for $\rho_{\varepsilon}$ holds,

$$
\rho_{\varepsilon}=\sum_{1 \leq \vec{n} \cdot \vec{\omega} \leq \alpha} a_{\vec{n}} \varepsilon^{\vec{n} \cdot \vec{\omega}}+o\left(\varepsilon^{[\alpha]}\right)
$$

where the coefficients can be calculated by the following recurrent formula:

$$
a_{\vec{e}_{1}}=-\frac{b_{\vec{e}_{1}, 0}}{b_{\overrightarrow{0}, 1}}
$$

and in general for $\vec{n}: 1<\vec{n} \cdot \vec{\omega} \leq \alpha$,

$$
\begin{aligned}
a_{\vec{n}}=-\frac{1}{b_{\overrightarrow{0}, 1}}\left(b_{\vec{n}, 0}\right. & +\sum_{\vec{p} \in \mathbf{R}^{\prime}{ }_{1}(\vec{n})} b_{\vec{n}-\vec{p}, 1} a_{\vec{p}} \\
& \left.+\sum_{i=2}^{n_{1}+n_{2}+\ldots+n_{k}} \sum_{\vec{p} \in \mathbf{R}_{i}(\vec{n})} b_{\vec{n}-\vec{p}, i}\left(\sum_{\vec{j}(\vec{p}) \in \mathbf{D}_{i}(\vec{p})} \prod_{\vec{r} \in \mathbf{R}^{\prime}{ }_{1}(\vec{p})} \frac{\left(a_{\vec{r}}\right)^{j_{\vec{r}}}}{\left(j_{\vec{r}}\right) !}\right)\right),
\end{aligned}
$$

where $\mathbf{D}_{i}(\vec{p})$ is the set of all nonnegative and integer solutions,

$$
\vec{j}(\vec{p}) \equiv\left(j_{\vec{r}}, \vec{r} \in \mathbf{R}_{1}^{\prime}(\vec{p})\right),
$$

for the Diophantine system

$$
\left\{\begin{array}{l}
\sum_{\vec{r} \in \mathbf{R}^{\prime}{ }_{1}(\vec{p})} j_{\vec{r}}=i, \\
\sum_{\vec{r} \in \mathbf{R}^{\prime}{ }_{1}(\vec{p})} \vec{r} \times j_{\vec{r}}=\vec{p} .
\end{array}\right.
$$

(ii) If the coefficients $b_{\vec{n}, 0}=0$ for all $\vec{n}$ satisfying $\vec{n} \cdot \vec{\omega} \leq \beta$ for some real number $\beta \in[1, \alpha]$, then $a_{\vec{n}}=0$ for all $\vec{n}$ satisfying $\vec{n} \cdot \vec{\omega} \leq \beta$.

(iii) If in addition condition $\mathbf{C}$ holds, then for any $0 \leq t_{\varepsilon} \rightarrow \infty$ balanced with $\varepsilon \rightarrow 0$ in such a way that $\varepsilon^{[\beta]} t_{\varepsilon} \rightarrow \lambda_{\beta} \in[0, \infty)$, where $\beta \in[1, \alpha]$ is a given real number, we have the following asymptotic relation:

$$
\exp \left\{\left(\sum_{1 \leq \vec{n} \cdot \vec{\omega}<[\beta]_{\vec{\omega}}} a_{\vec{n}} \varepsilon^{\vec{n} \cdot \vec{\omega}}\right) t_{\varepsilon}\right\} x_{\varepsilon}\left(t_{\varepsilon}\right) \rightarrow e^{-\lambda_{\beta} a^{(1)}} x_{0}(\infty) \quad \text { as } \varepsilon \rightarrow 0,
$$

where $a^{(1)}=a_{\vec{p}}$ with $\vec{p}=\vec{f}(\beta, \vec{\omega})$.

Remark 1 . The coefficients $a_{\vec{n}}, 1 \leq \vec{n} \cdot \vec{\omega} \leq \alpha$ can be calculated recursively from the recurrent formula (6) . By formula (6),$a_{\vec{n}}$ for a specific $\vec{n}$ depends on the set of coefficients $\left\{a_{\vec{p}}: \vec{p} \in \mathbf{R}_{1}^{\prime}(\vec{n})\right\} \subseteq\left\{a_{\vec{p}}: 1 \leq \vec{p} \cdot \vec{\omega}<\vec{n}\right\}$. Also by observing formulas (5) and (6), we find that the value of the coefficient $a_{\vec{n}}$ does not depend on the parameter vector $\vec{\omega}$ and the parameter $\alpha$. 
Remark 2. For a given parameter vector $\vec{\omega}$ and a given order $\alpha$, the expansion (5) of $\rho_{\varepsilon}$ takes a unique form, and so is the sequence of coefficients $a_{\vec{n}}, 1 \leq \vec{n} \cdot \vec{\omega}<\alpha$. Let all terms of the expansion (5) be ordered according to the asymptotic scale (3), i.e. these terms are sorted in ascending order in terms of the powers of $\varepsilon$. A natural choice of recursive algorithm would be to calculate the coefficients sequentially starting from the first term.

Remark 3. The theorem is consistent with the corresponding results in Silvestrov (1995, [10]) and Gyllenberg and Silvestrov (2008, 3]) for the reduced case $\vec{\omega}=1$ and $\alpha$ being a positive integer. If $\vec{\omega}$ is two-dimensional, i.e. $\vec{\omega}=(1, \omega)$ for some irrational $\omega>1$, and $\alpha \geq 1$ be some real number, Theorem 2 reduces in this case to the main result in a previous study by Ni, Silvestrov and Malyarenko (2008, 4]).

Remark 4. For illustration, we give the explicit expressions, calculated by formula (6), of the first few coefficients in the expansion (5) in terms of the given coefficients in $\mathbf{P}_{\vec{\omega}}^{(\alpha)}$ for the case $\vec{\omega}=(1, \sqrt{2}, \sqrt{3}), \alpha \geq 1+\sqrt{3}$. That is,

$$
\begin{gathered}
a_{(1,0,0)}=-\frac{b_{(1,0,0), 0}}{b_{(0,0,0), 1}} \quad a_{(0,1,0)}=-\frac{b_{(0,1,0), 0}}{b_{(0,0,0), 1}}, \quad a_{(0,0,1)}=-\frac{b_{(0,0,1), 0}}{b_{(0,0,0), 1}} \\
a_{(2,0,0)}=-\frac{1}{b_{(0,0,0), 1}}\left[b_{(2,0,0), 0}+b_{(1,0,0), 1} a_{(1,0,0)}+\frac{1}{2} b_{(0,0,0), 2} a_{(1,0,0)}^{2}\right] \\
a_{(1,1,0)}=-\frac{1}{b_{(0,0,0), 1}}\left[b_{(1,1,0), 0}+b_{(1,0,0), 1} a_{(0,1,0)}+b_{(0,1,0), 1} a_{(1,0,0)}\right. \\
\left.+b_{(0,0,0), 2} a_{(1,0,0)} a_{(0,1,0)}\right] .
\end{gathered}
$$

Note that the expressions of these coefficients do not depend on the exact values of the three components in $\vec{\omega}$. If $\vec{\omega}$ is another three-dimensional parameter vector, the corresponding coefficients $a_{(1,0,0)}, a_{(0,1,0)}, a_{(0,0,1)}, \ldots$, if included in the expansion for $\rho_{\varepsilon}$ up to order $\alpha$, will have exactly the same expressions as above. For larger values of $k$ and $\alpha$, it is better to program formula ([6). We refer to Ni (2010a, [5]) for a brief description of the computer implementation of formula (6).

\section{A Queueing EXAMPle}

Let us consider an initially empty $\mathrm{M} / \mathrm{G} / 1$ queueing system with FIFO discipline, working at a unit rate, having a Poisson customer flow with rate $\lambda$ and i.i.d. service times with a common distribution $G_{\varepsilon}(z)$ and a finite mean $\mu_{\varepsilon}=\int_{0}^{\infty} z G_{\varepsilon}(d z)$. Suppose that the service times have a perturbed gamma distribution $G_{\varepsilon}(z)$ with density

$$
g_{\varepsilon}\left(z ; \xi_{\varepsilon}, \theta\right)=z^{\xi_{\varepsilon}-1} \frac{e^{-z / \theta}}{\theta^{\xi_{\varepsilon}} \Gamma\left(\xi_{\varepsilon}\right)}, \quad z>0, \quad \xi_{\varepsilon}, \theta>0 .
$$

We assume that the shape parameter $\xi_{\varepsilon}$ takes the following form:

$$
\xi_{\varepsilon}=\xi_{0}-b_{1} \varepsilon^{\omega_{1}}+b_{2} \varepsilon^{\omega_{2}}+b_{3} \varepsilon^{\omega_{3}}+b_{4} \varepsilon^{2}+o\left(\varepsilon^{2}\right),
$$

where $\xi_{0} \geq \xi_{\varepsilon}, \omega_{1} \equiv 1, \omega_{2}, \omega_{3}$ are irrational numbers and $\omega_{2} / \omega_{3}$ is also an irrational number. For simplicity we assume $1<\omega_{2}<\omega_{3}<2$ and define the parameter vector $\vec{\omega}=\left(\omega_{1}=1, \omega_{2}, \omega_{3}\right)$. If the perturbation parameter $\varepsilon=0, g_{\varepsilon}\left(z ; \xi_{\varepsilon}, \theta\right)$ is reduced to $g_{0}\left(z ; \xi_{0}, \theta\right)$.

The first moment of $G_{\varepsilon}(z)$ is given as $\mu_{\varepsilon}=\mu_{0}-\theta b_{1} \varepsilon-\theta b_{2} \varepsilon^{\omega_{2}}-\theta b_{3} \varepsilon^{\omega_{3}}-\theta b_{4} \varepsilon^{2}+o\left(\varepsilon^{2}\right)$. By (8) we have $G_{\varepsilon}(z) \Rightarrow G_{0}(z)$ as $\varepsilon \rightarrow 0$ for every $z \geq 0$, and $\mu_{\varepsilon} \leq \mu_{0}$ but $\mu_{\varepsilon} \rightarrow \mu_{0}$ as $\varepsilon \rightarrow 0$. 
An important quantity is the occupation rate $\alpha_{\varepsilon}$ defined as the product of the customer arrival rate and the mean service time, i.e., $\alpha_{\varepsilon}=\lambda \mu_{\varepsilon}$. We impose the following condition on $\alpha_{0}$ :

D: $\alpha_{0}=1$.

The virtual waiting time $U_{\varepsilon t}$, also known as the workload, is defined as the time during which a customer would have to wait before getting service if he arrived at instant $t$. We are interested in the steady-state limit of the virtual waiting time process, $\left\{U_{\varepsilon t}, t \geq 0\right\}$ in the $\mathrm{M} / \mathrm{G} / 1$ queueing system with service time density (8).

By condition $\mathbf{D}, \alpha_{\varepsilon}=\lambda \mu_{\varepsilon}<\alpha_{0}=1$ for $\varepsilon>0$. In this case $U_{\varepsilon t} \rightarrow U_{\varepsilon}$ in distribution as $t \rightarrow \infty$ for some r.v. $U_{\varepsilon} \in[0, \infty]$. The object of interest is the steady-state limit for the tail distribution of the virtual waiting time, denoted by $\Psi_{\varepsilon}(u)=\mathrm{P}\left(U_{\varepsilon}>u\right)$.

For the special case $\varepsilon=0$ we have $\alpha_{0}=1$. It is known that in this case $U_{0}=\infty$ almost surely; hence $\Psi_{0}(u)=1$ for all $u \geq 0$. Our aim is to obtain the asymptotic behavior of $\Psi_{\varepsilon}(u)$ as $\varepsilon \rightarrow 0$ and $u \rightarrow \infty$ simultaneously but in some balanced manner. Note that $\alpha_{\varepsilon} \rightarrow 1$ as $\varepsilon \rightarrow 0$ and this is the interesting heavy traffic situation in queueing theory.

Due to a duality between the ruin probability of the classical risk process and the steady-state limit $\Psi_{\varepsilon}(u)$ of virtual waiting time for the $M / G / 1$ queue (see for example Asmussen 2000, 1]), and the fact that the ruin probability satisfies a defective renewal equation (Feller 1971, 2]), we know that under condition $\mathbf{D}, \Psi_{\varepsilon}(u)$ for $\varepsilon>0$ and as a function of $u$, satisfies the following perturbed renewal equation:

$$
\Psi_{\varepsilon}(u)=\alpha_{\varepsilon}\left(1-\widetilde{G}_{\varepsilon}(u)\right)+\alpha_{\varepsilon} \int_{0}^{u} \Psi_{\varepsilon}(u-s) \widetilde{G}_{\varepsilon}(d s), \quad u \geq 0,
$$

where $\widetilde{G}_{\varepsilon}(u)=\mu_{\varepsilon}^{-1} \int_{0}^{u}\left(1-G_{\varepsilon}(s)\right) d s, F_{\varepsilon}(u) \equiv \alpha_{\varepsilon} \widetilde{G}_{\varepsilon}(u)$.

Using condition $\mathbf{D}$, the defect and moments for $F_{\varepsilon}(u)$ take the forms

$$
\begin{gathered}
f_{\varepsilon}=\frac{\theta b_{1}}{\mu_{0}} \varepsilon+\frac{\theta b_{2}}{\mu_{0}} \varepsilon^{\omega_{2}}+\frac{\theta b_{3}}{\mu_{0}} \varepsilon^{\omega_{3}}+\frac{\theta b_{4}}{\mu_{0}} \varepsilon^{2}+o\left(\varepsilon^{2}\right), \\
m_{\varepsilon 1}=m_{01}-\frac{\theta^{2}}{2 \mu_{0}}\left(2 \xi_{0}+1\right) b_{1} \varepsilon+o(\varepsilon), \quad m_{\varepsilon 2}=m_{02}+o(\varepsilon),
\end{gathered}
$$

where $m_{0 r}, r \geq 2$, refers to the $r$ th moment of $F_{0}(u)$. Applying Theorem 2 yields the exponential asymptotic expansion for $\Psi_{\varepsilon}(u)$.

Theorem 3. Let $G_{\varepsilon}(u)$ be the service time distribution function with density (8) and $\varepsilon$ be the perturbation parameter. Also let condition $\boldsymbol{D}$ hold. Then:

(i) There exists a unique nonnegative solution, $\rho_{\varepsilon}$, of the characteristic equation (44), and the following asymptotic relation holds:

$$
\rho_{\varepsilon}=a_{(1,0,0)} \varepsilon+a_{(0,1,0)} \varepsilon^{\omega_{2}}+a_{(0,0,1)} \varepsilon^{\omega_{3}}+a_{(2,0,0)} \varepsilon^{2}+o\left(\varepsilon^{2}\right),
$$

where $a_{(1,0,0)}=\theta b_{1} / \mu_{0} m_{01}, a_{(0,1,0)}=\theta b_{2} / \mu_{0} m_{01}, a_{(0,0,1)}=\theta b_{3} / \mu_{0} m_{01}$, and

$$
a_{(2,0,0)}=\frac{\theta b_{4}}{m_{01} \mu_{0}}+\frac{\theta^{3} b_{1}^{2} \xi_{0}}{m_{01}^{2} \mu_{0}^{2}}+\frac{1}{2} \frac{\theta^{3} b_{1}^{2}}{m_{01}^{2} \mu_{0}^{2}}-\frac{1}{2} \frac{m_{02} \theta^{2} b_{1}^{2}}{m_{01}^{3} \mu_{0}^{2}} .
$$

(ii) For any $0 \leq u_{\varepsilon} \rightarrow \infty$ in such a way that $\varepsilon^{[\beta]_{\omega}} u_{\varepsilon} \rightarrow \lambda_{\beta} \in[0, \infty)$ for some $1 \leq \beta<\omega_{2}$, the following asymptotic relation holds:

$$
\Psi_{\varepsilon}\left(u_{\varepsilon}\right) \rightarrow \exp \left\{-\lambda_{\beta} \frac{\theta b_{1}}{\mu_{0} m_{01}}\right\} \quad \text { as } \varepsilon \rightarrow 0 .
$$


(iii) For any $0 \leq u_{\varepsilon} \rightarrow \infty$ in such a way that $\varepsilon^{[\beta]_{\omega}} u_{\varepsilon} \rightarrow \lambda_{\beta} \in[0, \infty)$ for some $\omega_{2} \leq \beta<\omega_{3}$, the following asymptotic relation holds:

$$
\exp \left\{\left(\frac{\theta b_{1}}{\mu_{0} m_{01}} \varepsilon\right) u_{\varepsilon}\right\} \Psi_{\varepsilon}\left(u_{\varepsilon}\right) \rightarrow \exp \left\{-\lambda_{\beta} \frac{\theta b_{2}}{\mu_{0} m_{01}}\right\} \quad \text { as } \varepsilon \rightarrow 0 .
$$

(iv) For any $0 \leq u_{\varepsilon} \rightarrow \infty$ in such a way that $\varepsilon^{[\beta]_{\omega}} u_{\varepsilon} \rightarrow \lambda_{\beta} \in[0, \infty)$ for some $\omega_{3} \leq \beta<2$, the following asymptotic relation holds:

$$
\exp \left\{\left(\frac{\theta b_{1}}{\mu_{0} m_{01}} \varepsilon+\frac{\theta b_{2}}{\mu_{0} m_{01}} \varepsilon^{\omega_{2}}\right) u_{\varepsilon}\right\} \Psi_{\varepsilon}\left(u_{\varepsilon}\right) \rightarrow \exp \left\{-\lambda_{\beta} \frac{\theta b_{3}}{\mu_{0} m_{01}}\right\} \quad \text { as } \varepsilon \rightarrow 0 .
$$

(v) For any $0 \leq u_{\varepsilon} \rightarrow \infty$ in such a way that $\varepsilon^{[\beta]_{\omega}} u_{\varepsilon} \rightarrow \lambda_{\beta} \in[0, \infty)$ for some $\beta=2$, the following asymptotic relation holds:

$$
\begin{aligned}
& \exp \left\{\left(\frac{\theta b_{1}}{\mu_{0} m_{01}} \varepsilon+\frac{\theta b_{2}}{\mu_{0} m_{01}} \varepsilon^{\omega_{2}}+\frac{\theta b_{3}}{\mu_{0} m_{01}} \varepsilon^{\omega_{3}}\right) u_{\varepsilon}\right\} \Psi_{\varepsilon}\left(u_{\varepsilon}\right) \\
& \rightarrow \exp \left\{-\lambda_{\beta} a_{(2,0,0)}\right\} \quad \text { as } \varepsilon \rightarrow 0,
\end{aligned}
$$

where $a_{(2,0,0)}$ is given in (i).

Remark 5. (i) The above asymptotic results also apply to the steady-state distribution of the actual waiting time, since the actual and the virtual waiting time have the same distribution in the steady state in the $M / G / 1$ queue. (ii) As mentioned before, there is a duality between the ruin probability of the classical risk process and the steady-state limit $\Psi_{\varepsilon}(u)$ of the virtual waiting time for the $M / G / 1$ queue, so the results above also have interpretations in risk theory.

\section{ProOFs}

\section{Proof of Theorem 2.}

Proof of statement (i). For all $\varepsilon$ small enough, conditions $\mathbf{A}$ and $\mathbf{B}$ imply that

(i) there exists a unique nonnegative solution $\rho_{\varepsilon}$ to the characteristic equation (4) and

(ii) $\rho_{\varepsilon} \rightarrow \rho_{0}=0$ as $\varepsilon \rightarrow 0$.

These small-enough values of $\varepsilon$ satisfy $\varepsilon \leq \varepsilon_{1}\left(\delta^{\prime}\right)$ for any choice of $\delta^{\prime} \in(0, \delta]$, and the corresponding solution satisfies $\rho_{\varepsilon} \leq \delta^{\prime}$. The proof can be found in Ni, Silvestrov, and Malyarenko (2008, 4]).

We are now in a position to do an asymptotic analysis of $\rho_{\varepsilon}$. Let us begin with taking Taylor's expansion for the function $e^{s \rho_{\varepsilon}}$ and obtain

$$
e^{s \rho_{\varepsilon}}=1+s \rho_{\varepsilon} / 1 !+\cdots+s^{[\alpha]} \rho_{\varepsilon}^{[\alpha]} /[\alpha] !+s^{[\alpha]+1} \rho_{\varepsilon}^{[\alpha]+1} e^{s \rho_{\varepsilon}} \theta_{\varepsilon,[\alpha], s} /([\alpha]+1) !,
$$

where $\theta_{\varepsilon,[\alpha], s}, s \in[0, \infty)$, is a continuous function in $s$ and $0 \leq \theta_{\varepsilon,[\alpha], s} \leq 1, s \in[0, \infty)$, holds.

Let us consider $\varepsilon \leq \varepsilon_{1}\left(\delta^{\prime}\right)$ for some fixed $\delta^{\prime} \in(0, \delta]$. From the discussions above we know that $\rho_{\varepsilon} \leq \delta^{\prime}$ for $\varepsilon \leq \varepsilon_{1}\left(\delta^{\prime}\right)$. Now integrating both sides of (14) and using condition $\mathbf{B}$ we have

$$
\int_{0}^{\infty} e^{s \rho_{\varepsilon}} F_{\varepsilon}(d s)=1-f_{\varepsilon}+m_{\varepsilon 1} \rho_{\varepsilon} / 1 !+\cdots+m_{\varepsilon[\alpha]} \rho_{\varepsilon}^{[\alpha]} /[\alpha] !+\rho_{\varepsilon}^{[\alpha]+1} M_{[\alpha]} \theta_{\varepsilon,[\alpha]},
$$

where

$$
M_{[\alpha]}=\frac{\sup _{\varepsilon \leq \varepsilon_{2}} \int_{0}^{\infty} s^{[\alpha]+1} e^{s \delta^{\prime}} F_{\varepsilon}(d s)}{([\alpha]+1) !}<\infty, \quad \theta_{\varepsilon,[\alpha]} \in[0,1] .
$$

Using (15), we can rewrite the characteristic equation (4) as

$$
m_{\varepsilon 1} \rho_{\varepsilon} / 1 !+\cdots+m_{\varepsilon[\alpha]} \rho_{\varepsilon}^{[\alpha]} /[\alpha] !+\rho_{\varepsilon}^{[\alpha]+1} M_{[\alpha]} \theta_{\varepsilon,[\alpha]}=f_{\varepsilon} .
$$


We shall at first show that there exists an asymptotic expansion (5). For the cases of $k=1$ and $k=2$, i.e. the parameter vector $\vec{\omega} \equiv 1$ or $\vec{\omega}=(1, \omega)$ in $\mathbf{P}_{\vec{\omega}}^{(\alpha)}$, the existence of this asymptotic expansion has been proved in Silvestrov (1995, 10]) and Ni, Silvestrov, Malyarenko (2008, 4]) respectively. To avoid needless repetition, we show that there exists an asymptotic expansion (5) for the case of $k>2$.

Because $\rho_{\varepsilon} \rightarrow 0$ as $\varepsilon \rightarrow 0$, the sum from the second to the last term in the left-hand side of the above equation (16) is $o\left(\rho_{\varepsilon}\right)$. Using condition $\mathbf{P}_{\vec{\omega}}^{(\alpha)}$, the first term on the right-hand side of the expansion for $f_{\varepsilon}$ is $-b_{\vec{e}_{1}, 0} \varepsilon$, i.e. of the order $O(\varepsilon)$. Also recall that $m_{\varepsilon r} \rightarrow m_{0 r} \in(0, \infty), r \geq 1$, and the first term in the expansion of $m_{\varepsilon 1}$ is $b_{\overrightarrow{0}, 1}$ by $\mathbf{P}_{\vec{\omega}}^{(\alpha)}$. Now let us divide both sides of (16) by $m_{\varepsilon 1} \varepsilon$ and using $\mathbf{P}_{\vec{\omega}}^{(\alpha)}$ we obtain $\rho_{\varepsilon} / \varepsilon \rightarrow-b_{\vec{e}_{1} 0} / b_{\overrightarrow{0}, 1}$, which implies the asymptotic relation (a) $\rho_{\varepsilon}=a_{\vec{e}_{1}} \varepsilon+\rho_{1 \varepsilon}, a_{\vec{e}_{1}}=-b_{\vec{e}_{1}, 0} / b_{\overrightarrow{0}, 1}, \rho_{1 \varepsilon}=o(\varepsilon)$. The asymptotic relation (a) reduces to (5) and (6) if the order parameter $\alpha$ satisfies $1 \leq \alpha<\left(\omega_{2} \wedge 2\right)$.

Recall that the asymptotic scale used in $\mathbf{P}_{\vec{\omega}}^{(\alpha)}$ is given by (3), where the parameter vector $\vec{\omega}$ satisfies the two properties. Obviously $\varepsilon^{\left(\omega_{2} \wedge 2\right)}$ is the next gauge function, in terms of the powers of $\varepsilon$, after $\varepsilon^{\omega_{1}}=\varepsilon$ in the sequence (3).

It follows from (a), condition $\mathbf{P}_{\vec{\omega}}^{(\alpha)}$, and inequalities $[\alpha] \leq[\alpha]_{\vec{\omega}} \leq \alpha<[\alpha]+1$ that equation (16) can be rewritten as

$$
m_{\varepsilon 1} \rho_{\varepsilon} / 1 !+\cdots+m_{\varepsilon[\alpha]} \rho_{\varepsilon}^{[\alpha]} /[\alpha] !+o\left(\varepsilon^{[\alpha]_{\vec{\omega}}}\right)=f_{\varepsilon} .
$$

Let us now consider $\omega_{2} \leq \alpha<2$ and assume $\omega_{i}>2$ for some $i$ where $2 \leq i \leq k$ but all $\omega_{j}$ with $j<i$ are less than 2 , say $\omega_{3}>2$ but $\omega_{2}<2$. We can continue the asymptotic analysis for this case. Substituting the expansions for $f_{\varepsilon}$ and $m_{\varepsilon r}$ from $\mathbf{P}_{\vec{\omega}}^{(\alpha)}$ into (17) and collecting the higher order terms, using at the same time relation (a), yields

$$
\left(b_{\overrightarrow{0}, 1}+o\left(\varepsilon^{\omega_{2}-1}\right)\right)\left(a_{\vec{e}_{1}} \varepsilon+\rho_{1 \varepsilon}\right)+o\left(\varepsilon^{\omega_{2}}\right)=-b_{\vec{e}_{1}, 0} \varepsilon-b_{\vec{e}_{2}, 0} \varepsilon^{\omega_{2}}-o\left(\varepsilon^{\omega_{2}}\right) .
$$

Substituting the formula for $a_{\vec{e}_{1}}$ from (a) into the above equation will make the lower order terms $-b_{\vec{e}_{1}, 0} \varepsilon$ on the two sides of the equation cancel each other, which gives

$$
o\left(\varepsilon^{\omega_{2}-1}\right)\left(a_{\vec{e}_{1}} \varepsilon\right)+\left(b_{\overrightarrow{0}, 1}+o\left(\varepsilon^{\omega_{2}-1}\right)\right) \rho_{1 \varepsilon}+o\left(\varepsilon^{\omega_{2}}\right)=-b_{\vec{e}_{2}, 0} \varepsilon^{\omega_{2}}-o\left(\varepsilon^{\omega_{2}}\right) .
$$

We can now divide both sides of (18) by $b_{\overrightarrow{0}, 1} \varepsilon^{\omega_{2}}$ and evaluate the corresponding limits. The asymptotic relation $\rho_{1 \varepsilon} / \varepsilon^{\omega_{2}} \rightarrow-b_{\vec{e}_{2}, 0} / b_{\overrightarrow{0}, 1}$ is then obtained, which is equivalent to the following, (b) $\rho_{1 \varepsilon}=a_{\vec{e}_{2}} \varepsilon^{\omega_{2}}+\rho_{2 \varepsilon}, a_{\vec{e}_{2}}=-b_{\vec{e}_{2}, 0} / b_{\overrightarrow{0}, 1}, \rho_{2 \varepsilon}=o\left(\varepsilon^{\omega_{2}}\right)$.

The asymptotic relations (a) and (b) provide formulas (5) and (6) for the case $\omega_{2} \leq$ $\alpha<2$ under the assumption $\omega_{3}>2$ but $\omega_{2}<2$. Note that these asymptotic relations remain valid even if $\alpha \geq 2$. Now let $\alpha=2$ and continue the asymptotic analysis in the same way. Using (a) and (b), (17) can be rewritten as

$$
\begin{aligned}
\left(b_{\overrightarrow{0}, 1}+\right. & \left.b_{\vec{e}_{1}} \varepsilon+o(\varepsilon)\right)\left(a_{\vec{e}_{1}} \varepsilon+a_{\vec{e}_{2}} \varepsilon^{\omega_{2}}+\rho_{2 \varepsilon}\right) \\
& +\left(b_{\overrightarrow{0}, 2}+o(1)\right)\left(a_{\vec{e}_{1}} \varepsilon+a_{\vec{e}_{2}} \varepsilon^{\omega_{2}}+\rho_{2 \varepsilon}\right)^{2} / 2+o\left(\varepsilon^{2}\right) \\
= & -b_{\vec{e}_{1}, 0} \varepsilon-b_{\vec{e}_{2}, 0} \varepsilon^{\omega_{2}}-b_{\vec{f}_{1}, 0} \varepsilon^{2}-o\left(\varepsilon^{2}\right),
\end{aligned}
$$

where $\vec{f}_{i}, i=1, \ldots, k$, denotes the vector with $i$ th component being equal to 2 .

Using the same technique as before, i.e. we first substitute the expression for the coefficients $a_{\vec{e}_{1}}$ and $a_{\vec{e}_{2}}$ into equation (19), then cancel terms of the order $\varepsilon$ and $\varepsilon^{\omega_{2}}$, divide the resulting equation by $\varepsilon^{2}$ and evaluate limits on both sides, the next term of 
the expansion for $\rho_{\varepsilon}$ is obtained as (c) $\rho_{2 \varepsilon}=a_{\vec{f}_{1}} \varepsilon^{2}+\rho_{3 \varepsilon}$,

$$
a_{\vec{f}_{1}}=-\frac{1}{b_{\overrightarrow{0}, 1}}\left(b_{\vec{f}_{1}, 0}+b_{\vec{e}_{1}, 1} a_{\vec{e}_{1}}+\frac{1}{2} b_{\overrightarrow{0}, 2} a_{\vec{e}_{1}}^{2}\right) \text {, }
$$

$\rho_{3 \varepsilon}=o\left(\varepsilon^{2}\right)$. The asymptotic relations (a), (b) and (c) together imply that relations (5) and (6) hold for the case: $\alpha=2$ and $\omega_{3}>2$ but $\omega_{2}<2$. The expression for $a_{\vec{f}_{1}}$ given above is exactly the same as given in formula (6).

The above asymptotic analysis is made under the assumption $\omega_{3}>2$ but $\omega_{2}<2$. For the more general case, i.e. $\omega_{i}>2$ for some $i$ where $2 \leq i \leq k$ but $\omega_{j}$ is less than 2 for any $j<i$, the asymptotic analysis follows the same line as above. For example if $\omega_{2}>2$, then we only have the asymptotic relation (a) for $\alpha<2$. If $\alpha=2$, by substituting (a) into (17), canceling then the lower order terms and finally dividing the resulting equation by $\varepsilon^{2}$, we can obtain the next term of the expansion for $\rho_{\varepsilon}$ which is of order $O\left(\varepsilon^{2}\right)$, where the corresponding expression for the coefficient $a_{\vec{f}_{1}}$ is the same as in (c). Or if it is $\omega_{4}>2$ and $\omega_{2}, \omega_{3}<2$ (assuming $k \geq 4$ ), after we have obtained (a), (b), the next step is to substitute them into (17) and divide the resulting equation by $\varepsilon^{\omega_{3}}$, by which we obtain the next term, of order $O\left(\varepsilon^{\omega_{3}}\right)$, in the expansion for $\rho_{\varepsilon}$. Finally for the last case $\omega_{2}, \ldots, \omega_{k}<2$, the asymptotic analysis is done in a similar manner as above.

Now suppose $\alpha>2$. We can continue the asymptotic analysis in the same way. Including sequentially higher order terms in the corresponding expansions in $\mathbf{P}_{\vec{\omega}}^{(\alpha)}$ and evaluating the corresponding limits in (17), we can show that $\rho_{\varepsilon}$ can be expanded to the form as in (5). This algorithm stops when the limits in (17) cannot be evaluated. This happens after we have expanded to the order of $O\left(\varepsilon^{[\alpha]_{\omega}}\right)$ for $\rho_{\varepsilon}$, since the next infinitesimal in the asymptotic scale (3) cannot be compared with the remaining terms in (17) which are of the form $o\left(\varepsilon^{[\alpha]_{\omega}}\right)$. Thus we have shown that $\rho_{\varepsilon}$ can be expanded to (5).

Provided that the asymptotic expansion (5) exists for $\rho_{\varepsilon}$ for $1 \leq k<\infty$, we can get the coefficients $a_{\vec{n}}$ in this expansion more effectively from the following formal asymptotic equality, which follows from equation (17):

$$
\begin{aligned}
\left(b_{\overrightarrow{0}, 1}+\sum_{1 \leq \vec{s} \cdot \vec{\omega} \leq \alpha-1} b_{\vec{s}, 1} \varepsilon^{\vec{s} \cdot \vec{\omega}}+o\left(\varepsilon^{[\alpha]_{\vec{\omega}}-1}\right)\right)\left(\sum_{1 \leq \vec{s} \cdot \vec{\omega} \leq \alpha} a_{\vec{s}} \varepsilon^{\vec{\varepsilon} \cdot \vec{\omega}}+o\left(\varepsilon^{[\alpha]_{\vec{\omega}}}\right)\right)+\cdots \\
\quad+\left(b_{\overrightarrow{0},[\alpha]}+o\left(\varepsilon^{[\alpha]_{\vec{\omega}}-[\alpha]}\right)\right) \frac{1}{[\alpha] !}\left(\sum_{1 \leq \vec{s} \cdot \vec{\omega} \leq \alpha} a_{\vec{s} \cdot \varepsilon^{\vec{s} \cdot \vec{\omega}}}+o\left(\varepsilon^{[\alpha]_{\vec{\omega}}}\right)\right)^{[\alpha]}+o\left(\varepsilon^{[\alpha]_{\vec{\omega}}}\right) \\
=-\left(\sum_{1 \leq \vec{s} \cdot \vec{\omega} \leq \alpha} b_{\vec{s}, 0} \varepsilon^{\vec{s} \cdot \vec{\omega}}+o\left(\varepsilon^{[\alpha]^{\omega}}\right)\right) .
\end{aligned}
$$

The coefficients $a_{\vec{n}}$ for $1 \leq \vec{n} \cdot \vec{\omega} \leq \alpha$ can be calculated by equating the coefficients for terms of the same order in the left-hand and right-hand sides of (20), which will yield formula (6). The right-hand side in (20) contributes to the first term in the brackets, i.e. $b_{\vec{n}, 0}$, in (6). In the left-hand side of (20), the first product of sums contributes to the second term, i.e. $\sum b_{\vec{n}-\vec{p}, 1} a_{\vec{p}}$, in (6). Finally the third term in formula (6) reflects the contribution of all the rest of the products of sums in the left-hand side of (20). For example, in the sum with index $2 \leq i \leq n_{1}+\cdots+n_{k}$ in (6) , the summand $\sum b_{\vec{n}-\vec{p}, i}(\cdots)$ represents the contribution of $\left(b_{\overrightarrow{0}, i}+\cdots\right) \times\left(\sum a_{\vec{s}} \varepsilon^{\vec{s} \cdot \vec{\omega}}+\cdots\right)^{i} / i$ ! in the left-hand side of (20). 
Using (20) we obtain $a_{\vec{e}_{1}}=-b_{\vec{e}_{1}, 0} / b_{\overrightarrow{0}, 1}$, which is consistent with formula (6). To prove that using (20) to calculate the coefficient coincides with using formula (6), it suffices to show that this is true for an arbitrary coefficient $a_{\vec{n}}$, where $\vec{n}$ satisfies $1<\vec{n} \cdot \vec{\omega} \leq \alpha$.

By collecting terms with higher orders than $\varepsilon^{\vec{n} \cdot \vec{\omega}}$, the first product of sums to the left-hand side in (20) can be rewritten as

$$
\sum_{1 \leq \vec{p} \cdot \vec{\omega}<\vec{n} \cdot \vec{\omega}} \sum_{\vec{s} \in \mathbf{R}_{1}(\vec{p})} b_{\vec{p}-\vec{s}, 1} a_{\vec{s}} \varepsilon^{\vec{p} \cdot \vec{\omega}}+\underbrace{\sum_{\vec{p} \in \mathbf{R}_{1}(\vec{n})} b_{\vec{n}-\vec{p}, 1} a_{\vec{p}} \varepsilon^{\vec{n} \cdot \vec{\omega}}}_{(*)}+o\left(\varepsilon^{\vec{n} \cdot \vec{\omega}}\right),
$$

where the set $\mathbf{R}_{1}(\cdot)$ is defined in Section 2 . Note that for some $\vec{s}$ such that $\vec{s} \notin \mathbf{R}_{1}(\vec{q})$, the corresponding $b_{\vec{q}-\vec{s}, 1} a_{\vec{s}} \varepsilon^{\vec{q} \cdot \vec{\omega}}$ is not a valid term that can be generated by the first product of sums to the left-hand side of (20). This justifies the use of $\mathbf{R}_{1}(\cdot)$ in (21).

In (21),$(*)$ represents the terms that are of order $O\left(\varepsilon^{\vec{n} \cdot \vec{\omega}}\right)$ and is obviously the only relevant term for calculating the coefficient $a_{\vec{n}}$. Let us rewrite it in a more convenient form:

$$
(*)=b_{\overrightarrow{0}, 1} a_{\vec{n}} \varepsilon^{\vec{n} \cdot \vec{\omega}}+\sum_{\vec{s} \in \mathbf{R}^{\prime}{ }_{1}(\vec{n})} b_{\vec{n}-\vec{s}, 1} a_{\vec{s}} \varepsilon^{\vec{n} \cdot \vec{\omega}},
$$

where $\mathbf{R}^{\prime}{ }_{1}(\vec{n}) \equiv \mathbf{R}_{1}(\vec{n}) \backslash\{\vec{n}\}$ as defined in Section 2. Expression (22) shows the contribution of the first product of sums in the left-hand side of (20). Now let us consider the $i$ th, $2 \leq i \leq[\vec{n} \cdot \vec{\omega}]$, product of sums, i.e. $\left(b_{\overrightarrow{0}, i}+\cdots\right) \times\left(\sum a_{\vec{s}} \varepsilon^{\vec{s} \cdot \vec{\omega}}+\cdots\right)^{i} / i$ !, to the left-hand side in (20). The latter product of sums, i.e. for $[\vec{n} \cdot \vec{\omega}]<i \leq[\alpha]$, can be obviously ignored for calculating $a_{\vec{n}}$ since it is of the order $o\left(\varepsilon^{\vec{n} \cdot \vec{\omega}}\right)$.

For simplicity, we consider only the terms of the order $O\left(\varepsilon^{\vec{n} \cdot \vec{\omega}}\right)$, since the coefficient $a_{\vec{n}}$ is determined only by those terms. We collect all terms in the $i$ th product of sums in (20) such that $\left(b_{\overrightarrow{0}, i}+\cdots\right)$ contributes $b_{\vec{n}-\vec{p}} \varepsilon^{(\vec{n}-\vec{p}) \vec{\omega}}$ and $\left(\sum a_{\vec{s}} \varepsilon^{\vec{s} \cdot \vec{\omega}}+\cdots\right)^{i} / i$ ! contributes $a_{\vec{p}} \varepsilon^{\vec{p} \cdot \vec{\omega}}$ for all $\vec{p}$ in the set $\widetilde{\mathbf{R}}_{i}(\vec{n})=\left\{\vec{p}: p_{1} \leq n_{1}, \ldots, p_{k} \leq n_{k}, \vec{p} \cdot \vec{\omega} \geq i\right\}$, where $\vec{n}, \vec{p} \in \mathbf{N}_{\mathbf{0}}^{\mathbf{k}}$.

The use of the set $\widetilde{\mathbf{R}}_{i}(\vec{n})$ is justified by the facts: (i) if $p_{j} \geq n_{j}$ for any $j=1, \ldots, k$, $b_{\vec{n}-\vec{p}} \varepsilon^{(\vec{n}-\vec{p}) \cdot \vec{\omega}}$ is not a valid term in $\left(b_{\overrightarrow{0}, i}+\cdots\right)$; (ii) the term with minimum order of $\varepsilon$ produced by $\left(\sum a_{\vec{s}} \varepsilon^{\vec{s} \cdot \vec{\omega}}+\cdots\right)^{i} / i$ ! is $\varepsilon^{i}$ which implies $\vec{p} \cdot \vec{\omega} \geq i$.

Therefore the $i$ th product of sums in equation (20), i.e. $\left(b_{\overrightarrow{0}, i}+\cdots\right) \times\left(\sum a_{\vec{s}} \varepsilon^{\vec{s} \cdot \vec{\omega}}+\cdots\right)^{i} / i$ !, produces the following terms that are of the order $O\left(\varepsilon^{\vec{n} \cdot \vec{\omega}}\right)$,

$$
\varepsilon^{\vec{n} \cdot \vec{\omega}} \sum_{\vec{p} \in \widetilde{\mathbf{R}}_{i}(\vec{n})} b_{\vec{n}-\vec{p}, i}\left(\sum_{\vec{j}(\vec{p}) \in \widetilde{\mathbf{D}}_{i}(\vec{p})} \prod_{1 \leq \vec{r} \cdot \vec{\omega} \leq \vec{p} \cdot \vec{\omega}} \frac{\left(a_{\vec{r}}\right)^{j_{\vec{r}}}}{\left(j_{\vec{r}}\right) !}\right),
$$

where $\widetilde{\mathbf{D}}_{i}(\vec{p})$ is the set of all nonnegative integer solutions $\vec{j}(\vec{p}) \equiv\left(j_{\vec{r}}, 1 \leq \vec{r} \cdot \vec{\omega} \leq \vec{p} \cdot \vec{\omega}\right)$ of the system

$$
\left\{\begin{array}{l}
\sum_{(1 \leq \vec{r} \cdot \vec{\omega} \leq \vec{p} \cdot \vec{\omega})} j_{\vec{r}}=i \\
\sum_{(1 \leq \vec{r} \cdot \vec{\omega} \leq \vec{p} \cdot \vec{\omega})} \vec{r} \times j_{\vec{r}}=\vec{p}
\end{array}\right.
$$

Note that the combinatoric counting of the coefficients in the product $\prod\left(a_{\vec{r}}\right)^{j_{\vec{r}}}$ yields the number $i ! /\left(\prod\left(j_{\vec{r}}\right) !\right)$. But the coefficient $i$ ! cancels with the denominator of

$$
\left(\sum a_{\vec{s}} \varepsilon^{\vec{s} \cdot \vec{\omega}}+\cdots\right)^{i} / i !
$$


Now consider system (24). The second equation leads to $k$ equations since the dimension of vectors $\vec{r}$ and $\vec{p}$ is $k$, and adding these $k$ equations yields

$$
\sum_{(1 \leq \vec{r} \cdot \vec{\omega} \leq \vec{p} \cdot \vec{\omega})}\left(\sum_{l=1}^{k} r_{l}\right) \times j_{\vec{r}}=\sum_{l=1}^{k} p_{l} .
$$

Because $\sum_{l=1}^{k} r_{l} \geq 1$, we have $\sum_{(1 \leq \vec{r} \cdot \vec{\omega} \leq \vec{p} \cdot \vec{\omega})}\left(\sum_{l=1}^{k} r_{l}\right) \times j_{\vec{r}} \geq \sum_{(1 \leq \vec{r} \cdot \vec{\omega} \leq \vec{p} \cdot \vec{\omega})} j_{\vec{r} .}$. This fact, together with the first equation in system (24), implies that $\sum_{l=1}^{k} p_{l} \geq i$ must be satisfied in order to make system (24) consistent and so to have solutions. Because of this remark and also the fact that $\sum_{l=1}^{k} p_{l} \geq i \Rightarrow \vec{p} \cdot \vec{\omega} \geq i$, we can reduce the set $\widetilde{\mathbf{R}}_{i}(\vec{n})$ in expression (23) to the set $\mathbf{R}_{i}(\vec{n})$.

Moreover, the set $\{\vec{r}: 1 \leq \vec{r} \cdot \vec{\omega} \leq \vec{p} \cdot \vec{\omega}\}$ in system (24) can be reduced to the smaller set $\mathbf{R}^{\prime}{ }_{1}(\vec{p})$. The arguments are as follows. Consider an arbitrary $\vec{r}$ from the set

$$
\{\vec{r}: 1 \leq \vec{r} \cdot \vec{\omega} \leq \vec{p} \cdot \vec{\omega}\} .
$$

If $\vec{r}=\vec{p}$, then in order to make the second equation in (24) satisfied, $j_{\vec{r}}$ must take the value one and all the remaining coefficients $j_{\vec{s}}, 1 \leq \vec{s} \cdot \vec{\omega}<\vec{p} \cdot \vec{\omega}$ must equal zero. In this case, the left-hand side of the first equation in (24) must equal one, which contradicts the fact that the right-hand side is greater than or equal to two, i.e. $i \geq 2$. So $\vec{p}$ can be reduced from the set. Further, if any component of $\vec{r}$, say the $i$ th component, satisfies $r_{i}>p_{i}$, then $j_{\vec{r}}$ must be zero; otherwise the second equation in system (24) does not have any solutions. But when $j_{\vec{r}}=0$, we have $\left(a_{\vec{r}}\right)^{j_{\vec{r}}} /\left(j_{\vec{r}}\right) !=1$, which can be ignored in the product

$$
\prod \frac{\left(a_{\vec{r}}\right)^{j_{\vec{r}}}}{\left(j_{\vec{r}}\right) !}
$$

Therefore, all vectors which contain at least one component greater than the corresponding component of $\vec{p}$ can be reduced from the set $\{\vec{r}: 1 \leq \vec{r} \cdot \vec{\omega} \leq \vec{p} \cdot \vec{\omega}\}$. Finally $\sum_{l=1}^{k} r_{l} \geq 1$ should hold; otherwise $\vec{r}$ can be $\overrightarrow{0}$ and as a consequence $a_{\overrightarrow{0}}$ is included in expression (23), but $a_{\overrightarrow{0}}$ does not exist in the expansion of $\rho_{\varepsilon}$. Hence, the set $\{\vec{r}: 1 \leq \vec{r} \cdot \vec{\omega} \leq \vec{p} \cdot \vec{\omega}\}$ in system (24) can be reduced to the set $\mathbf{R}_{1}^{\prime}(\vec{p})$.

All the remarks above imply that (23) and (24) can be reduced to

$$
\varepsilon^{\vec{n} \cdot \vec{\omega}} \sum_{\vec{p} \in \mathbf{R}_{i}(\vec{n})} b_{\vec{n}-\vec{p}, i}\left(\sum_{\vec{j}(\vec{p}) \in \mathbf{D}_{i}(\vec{p})} \prod_{\vec{r} \in \mathbf{R}^{\prime}(\vec{p})} \frac{\left(a_{\vec{r}}\right)^{j_{\vec{r}}}}{\left(j_{\vec{r}}\right) !}\right),
$$

where $\mathbf{D}_{i}(\vec{p})$ is the set of all nonnegative integer solutions $\vec{j}(\vec{p}) \equiv\left(j_{\vec{r}}, \vec{r} \in \mathbf{R}^{\prime}{ }_{1}(\vec{p})\right)$ of the system (7).

Having considered the terms of order $O\left(\varepsilon^{\vec{n} \cdot \vec{\omega}}\right)$ in the left-hand side of (20), we now collect the terms of order $O\left(\varepsilon^{\vec{n} \cdot \vec{\omega}}\right)$ in the right-hand side of (20), which gives simply $b_{\vec{n}, 0} \varepsilon^{\vec{n} \cdot \vec{\omega}}$. Due to this fact and also (22) and (25), we obtain the following recurrence formula:

$$
\begin{aligned}
a_{\vec{n}}=-\frac{1}{b_{\overrightarrow{0}, 1}}\left(b_{\vec{n}, 0}+\right. & \sum_{\vec{p} \in \mathbf{R}^{\prime}{ }_{1}(\vec{n})} b_{\vec{n}-\vec{p}, 1} a_{\vec{p}} \\
& +\underbrace{\sum_{i=2}^{[\vec{n} \cdot \vec{\omega}]}}_{(* *)} \sum_{\vec{p} \in \mathbf{R}_{i}(\vec{n})} b_{\vec{n}-\vec{p}, i}\left(\sum_{\vec{j}(\vec{p}) \in \mathbf{D}_{i}(\vec{p})} \prod_{\vec{r} \in \mathbf{R}^{\prime}{ }_{1}(\vec{p})} \frac{\left(a_{\vec{r}}\right)^{j_{\vec{r}}}}{\left(j_{\vec{r}}\right) !}\right)),
\end{aligned}
$$

where $\mathbf{D}_{i}(\vec{p})$ is the set defined above. 
As mentioned before, only the first up to the $i$ th, $i=[\vec{n} \cdot \vec{\omega}]$, product of sums are relevant for calculating $a_{\vec{n}}$; all later products of sums are obviously of order $o\left(\varepsilon^{\vec{n} \cdot \vec{\omega}}\right)$ and can be ignored. Therefore $(* *)$ in formula (26) tells us that we should sum from $i=2$ to $i=[\vec{n} \cdot \vec{\omega}]$. However, $(* *)$ can be reduced to summing from $i=2$ to $i=\sum_{j=1}^{k} n_{j}$. To see this, note that if $i=\sum_{j=1}^{k} n_{j}+m$ in (26), where $m$ is an integer and $m \geq 1$, we have

$$
\begin{aligned}
\mathbf{R}_{i}(\vec{n}) & =\left\{\vec{p}: p_{1} \leq n_{1}, \ldots, p_{k} \leq n_{k}, \sum_{j=1}^{k} p_{j} \geq i\right\} \\
& \subseteq\left\{\vec{p}: p_{1} \leq n_{1}, \ldots, p_{k} \leq n_{k}, \sum_{j=1}^{k} p_{j}>\sum_{j=1}^{k} n_{j}\right\}=\varnothing .
\end{aligned}
$$

In this case $\vec{p} \in \mathbf{R}_{i}(\vec{n})=\varnothing$, which implies that the third term in the sum in (26) is equal to zero. Therefore formula (26) can be reduced to the recurrence formula (6), and the proof of statement (i) of Theorem 2 is now complete.

Proof of statement (ii). This statement is a direct corollary of the recursive formula (6).

Proof of statement (iii). This statement can be proved by applying Theorem 1. Under the balancing condition $\varepsilon^{[\beta]_{\omega}} t_{\varepsilon} \rightarrow \lambda_{\beta} \in[0, \infty)$ we have, as $\varepsilon \rightarrow 0, o\left(\varepsilon^{[\beta]_{\omega}}\right) t_{\varepsilon} \rightarrow 0$ implying $\exp \left\{o\left(\varepsilon^{[\beta]_{\omega}}\right) t_{\varepsilon}\right\} \rightarrow 1$. Also $a^{(1)} \varepsilon^{[\beta]_{\omega}} t_{\varepsilon} \rightarrow a^{(1)} \lambda_{\beta}$. Due to these facts, one can easily transform the asymptotic relation from Theorem 1 into the asymptotic relation given in statement (iii).

III. Proof of Theorem 3. To apply Theorem 2, we need to show that conditions A-C hold. Using condition $\mathbf{D}$, the distribution function generating the renewal equation (10), $F_{\varepsilon}(u)$, takes the form

$$
F_{\varepsilon}(u)=\alpha_{\varepsilon} \widetilde{G}_{\varepsilon}(u)=\frac{1}{\mu_{0}} \int_{0}^{u}\left(1-G_{\varepsilon}(s)\right) d s .
$$

Condition A holds because $G_{\varepsilon}(u) \rightarrow G_{0}(u)$ as $\varepsilon \rightarrow 0$ due to (8), (9) and $G_{0}(u)$ is obviously proper and nonarithmetic. Using integration by parts we have for $\delta<\frac{1}{\theta}$ that

$$
\int_{0}^{\infty} e^{\delta s} F_{\varepsilon}(d s)=-\frac{1}{\mu_{0} \delta}+\frac{1}{\mu_{0} \delta} \int_{0}^{\infty} e^{\delta s} s^{\xi_{\varepsilon}-1} \frac{e^{-s / \theta}}{\theta_{\varepsilon} \Gamma\left(\xi_{\varepsilon}\right)} d s<\infty
$$

which implies that condition $\mathbf{B}$ is satisfied for $\delta \in(0,1 / \theta)$.

Let us consider the forcing function $q_{\varepsilon}(u)=\alpha_{\varepsilon}\left(1-\widetilde{G}_{\varepsilon}(u)\right)=\alpha_{\varepsilon}-F_{\varepsilon}(u)$. It follows from the formula for $F_{\varepsilon}(u)$ and the fact $\alpha_{\varepsilon} \rightarrow \alpha_{0}=1$ that $q_{\varepsilon}(u)$ converges pointwise to $q_{0}(u)=1-F_{0}(u)$ as $\varepsilon \rightarrow 0$ and for all $u \geq 0$. These facts and the monotonicity of the function $q_{\varepsilon}(u)$ imply that $q_{\varepsilon}(u)$ converges locally uniformly to $q_{0}(u)$ almost everywhere on the set of continuity of the limit function $q_{0}(u)$, i.e. on $[0, \infty)$. Thus condition $\mathbf{C}(\mathbf{a})$ holds. Condition $\mathbf{C}(\mathbf{b})$ obviously holds since $0 \leq q_{\varepsilon}(u) \leq 1$. Similar to what we did in (27), we can apply integration by parts twice and get for $\gamma \in\left(0, \frac{1}{\theta}\right)$ that

$$
\varlimsup_{0 \leq \varepsilon \rightarrow 0} \int_{0}^{\infty} e^{\gamma s}\left(1-\widetilde{G}_{\varepsilon}(s)\right) d s<\infty .
$$


Hence we have for any $\gamma \in\left(0, \frac{1}{\theta}\right)$ that

$$
\begin{gathered}
\varlimsup_{0 \leq \varepsilon \rightarrow 0} h \sum_{r \geq T / h} \sup _{r h \leq t \leq(r+1) h} e^{\gamma t}\left|q_{\varepsilon}(t)\right| \leq \varlimsup_{0 \leq \varepsilon \rightarrow 0} h \sum_{r \geq T / h} e^{\gamma(r+1) h} \alpha_{\varepsilon}\left(1-\widetilde{G}_{\varepsilon}(r h)\right) \\
\leq \varlimsup_{0 \leq \varepsilon \rightarrow 0} e^{\gamma h} \int_{T-h}^{\infty} e^{\gamma s}\left(1-\widetilde{G}_{\varepsilon}(s)\right) d s \rightarrow 0 \quad \text { as } T \rightarrow \infty,
\end{gathered}
$$

which implies that condition $\mathbf{C}$ (c) holds.

Finally we check condition $\mathbf{P}_{\vec{\omega}}^{(\alpha)}$. Using the assumption $\alpha_{0}=1$, the defect for $F_{\varepsilon}(u)$, $f_{\varepsilon}$, has the following asymptotic expansion:

$$
f_{\varepsilon}=1-\frac{\mu_{\varepsilon}}{\mu_{0}}=\frac{\theta b_{1}}{\mu_{0}} \varepsilon+\frac{\theta b_{2}}{\mu_{0}} \varepsilon^{\omega_{2}}+\frac{\theta b_{3}}{\mu_{0}} \varepsilon^{\omega_{3}}+\frac{\theta b_{4}}{\mu_{0}} \varepsilon^{2}+o\left(\varepsilon^{2}\right),
$$

and the moments for $F_{\varepsilon}(u)$ take the following form:

$$
m_{\varepsilon r}=\frac{\mu_{\varepsilon}^{(r+1)}}{\mu_{0}(r+1)}=\frac{\theta^{r}\left(\xi_{\varepsilon}+r-1\right) \cdots \xi_{\varepsilon}}{\mu_{0}(r+1)}, \quad r \geq 1,
$$

where $\mu_{\varepsilon}^{(r+1)}$ is the $(r+1)$ th moment of $G_{\varepsilon}(z)$. The notation $\left(\xi_{\varepsilon}+r-1\right) \cdots \xi_{\varepsilon}$ is understood as: $\left(\xi_{\varepsilon}+r-1\right) \cdots \xi_{\varepsilon}=\xi_{\varepsilon}$ if $r=1,\left(\xi_{\varepsilon}+r-1\right) \cdots \xi_{\varepsilon}=\left(\xi_{\varepsilon}+1\right) \xi_{\varepsilon}$ if $r=2$ and so on.

We can rewrite the formulas for $f_{\varepsilon}$ and $m_{\varepsilon r}$ in the form of $\mathbf{P}_{\vec{\omega}}^{(\alpha)}$ for $\alpha=2$ and for any given $\vec{\omega}$, which yields (11) and (12). Now applying Theorem 2 (i) we obtain the asymptotic expansion (13) for $\rho_{\varepsilon}$. Using Theorem 2 (iii) we can prove the statements (ii) $-(\mathbf{v})$ of Theorem 3 .

\section{BIBLIOGRAPHY}

1. S. Asmussen, Ruin Probabilities, World Scientific, Singapore, 2000. MR 1794582(2001m:62119)

2. W. Feller, An Introduction to Probability Theory and its Applications, vol. II, Wiley, New York, 1971. MR.0270403(42:5292)

3. M. Gyllenberg and D. S. Silvestrov, Quasi-stationary Phenomena in Nonlinearly Perturbed Stochastic Systems, De Gruyter Expositions in Mathematics, vol. 44, Walter de Gruyter, Berlin, 2008. MR 2456816 (2009k:60005)

4. Y. Ni, D. Silvestrov, and A. Malyarenko, Exponential asymptotics for nonlinearly perturbed renewal equation with non-polynomial perturbations, J. Numer. Appl. Math. 1(96) (2008), 173-197.

5. Y. Ni, Analytical and numerical studies of perturbed renewal equations with multivariate nonpolynomial perturbations, Journal of Applied Quantitative Methods 5(3) (2010a), 498-515.

6. Y. Ni, Perturbed Renewal Equations with Non-polynomial Perturbations, Licentiate Thesis, Mälardalen University, 2010b.

7. D. S. Silvestrov, A generalization of the renewal theorem, Dokl. Akad. Nauk. Ukr. SSR, Ser. A, 11 (1976), 978-982. MR0483057 (58:3086)

8. D. S. Silvestrov, The renewal theorem in a series scheme. I, Teor. Veroyatn. Mat. Stat. 18 (1978), 144-161; English transl. in Theory Probab. Math. Statist. 18, 155-172. MR0488350 $(58: 7899)$

9. D. S. Silvestrov, The renewal theorem in a series scheme. II, Teor. Veroyatn. Mat. Stat. 20 (1979), 97-116; English transl. in Theory Probab. Math. Statist. 20, 113-130. MR529265 (80g:60092)

10. D. S. Silvestrov, Exponential asymptotic for perturbed renewal equations, Teor. İmovirn. Mat. Stat. 52 (1995), 143-153; English transl. in Theory Probab. Math. Statist. 52, 153-162. MR $1445549(97 \mathrm{~m}: 60127)$

Division of Applied Mathematics, School of Education, Culture, and Communication, Mälardalen University, VÄSTERÅs 721 23, SwEdEN

E-mail address: ying.ni@mdh.se 